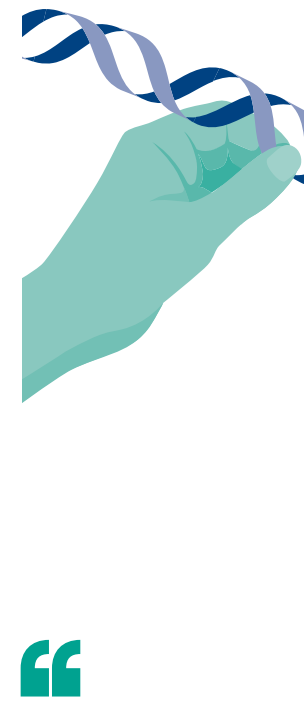

dystrophin

expression in a

wide range of

muscles...

improved

muscle function,

reduced cardiac

arrhythmogenic

susceptibility

and prolonged

survival

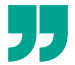

\title{
Dual apoC-II mimetic and apoC-III antagonist for hypertriglyceridaemia
}

An apolipoprotein C-II (apoC-II) mimetic peptide known as D6PV lowers triglyceride levels by activating lipoprotein lipase (LPL) and antagonizing the triglyceride-raising effects of apoC-III, according to a new study published in Science Translational Medicine. D6PV increases triglyceride lipolysis in mouse models of hypertriglyceridaemia (HTG) and shows good bioavailability in non-human primates, indicating its potential as a treatment for patients with HTG.

Given that patients with elevated plasma triglyceride levels are at increased risk of cardiovascular disease, novel therapies targeting HTG are needed. Anna Wolska (NHLBI, USA) and colleagues designed a peptide called D6PV that has dual apoC-II mimetic and apoC-III antagonist activity. "The inspiration for designing the D6PV peptide was from a simulation on the Anton 2 supercomputer, which was specifically created to perform 30 molecular dynamics simulations," comments senior investigator Alan Remaley (NHLBI, USA).

The efficacy of D6PV was first tested in plasma from humans with HTG. D6PV was a more potent ex vivo activator of lipolysis than full-length apoC-II in plasma from patients with apoC-II deficiency and also increased LPL-dependent lipolysis in plasma from patients with moderate HTG. To test the hypothesis that this effect might be associated with antagonism of apoC-III, LPL was added to pooled human HTG plasma and incubated with D6PV. Addition of apoC-III to human HTG plasma blunted the lipolysis of triglycerides, but D6PV prevented this inhibition by displacing apoC-III from lipoproteins.

In apoC-II-deficient mice, intraperitoneal delivery of D6PV resulted in a rapid reduction in plasma triglyceride levels by $70 \%$ after $1 \mathrm{~h}$ and by $85 \%$ after $3 \mathrm{~h}$. Furthermore, transgenic mice expressing human APOC3 (hAPOC3-Tg mice) treated with D6PV had an $80 \%$ decrease in plasma triglyceride levels after $3 \mathrm{~h}$ and an $85 \%$ reduction in total plasma apoC-III levels, in addition to reduced apoC-III bound to VLDL, LDL and HDL particles. D6PV also reduced LDL levels by $10 \%$ in hAPOC $3-\mathrm{Tg}$ mice lacking the LDL receptor. These findings support a mechanism whereby D6PV can lower triglyceride levels by displacing apoC-III from triglyceride-rich lipoproteins, independently of its direct effect of stimulating LPL.

Finally, the bioavailability of D6PV was tested in both mice and non-human primates. D6PV had a subcutaneous bioavailability of $80 \%$ and an extended terminal half-life of $42-50 \mathrm{~h}$ in non-human primates, which translates to a half-life of 3-4 days in humans using allometric scaling.

"Our research is still in early stages, but it is possible that this investigational therapy could be developed as a once-a-week injectable chronic therapy for apoC-Il-deficient patients or patients with HTG," highlights senior investigator Matt Devalaraja (Corvidia Therapeutics, USA). "We look forward to continuing our research on D6PV and our partnership with the NHLBI."

Karina Huynh

disputed

or limited

evidence for

causation of

LOTS should

not be rou-

tinely tested

for diagnostic

purposes incorrect diagnosis in a patient and their family members. Many of us have seen numerous cases of wrongly diagnosed patients based on misinterpreted genetics, many of whom have suffered undue mental health and physical consequences because of these errors."

Gregory B. Lim

ORIGINAL ARTICLE Adler, A. et al. An international, multicentered, evidence-based reappraisal of genes reported to cause congenital long QT syndrome. Circulation https://doi.org/10.1161/CIRCULATIONAHA. 119.043132 (2020)

RELATED ARTICLE Offerhaus, J. A. et al.

Epidemiology of inherited arrhythmias. Nat. Rev.

Cardiol. https://doi.org/10.1038/s41569-019-

0266-2 (2019)
ORIGINAL ARTICLE Wolska, A. et al. A dual apolipoprotein C-II mimetic-apolipoprotein C-III antagonist peptide lowers plasma triglycerides. Sci. Transl Med. 12, eaaw7905 (2020)

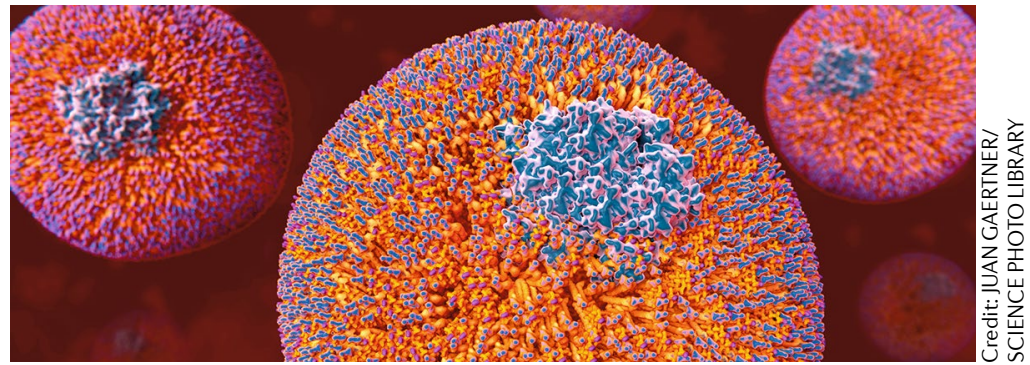

